Asymptotic Symmetry and its Implication in Elementary Particle Physics 
This page is intentionally left blank 


\section{Asymptotic Symmetry and its Implication in Elementary Particle Physics}

\section{S. Oneda}

University of Maryland

\section{Y. Koide}

University of Shizuoka 
Published by

World Scientific Publishing Co. Pte. Ltd.

P O Box 128, Farrer Road, Singapore 9128

USA office: Suite 1B, 1060 Main Street, River Edge, NJ 07661

UK office: 73 Lynton Mead, Totteridge, London N20 8DH

\section{ASYMPTOTIC SYMMETRY AND ITS IMPLICATION IN ELEMENTARY PARTICLE PHYSICS}

Copyright @ 1991 by World Scientific Publishing Co. Pte. Ltd.

All rights reserved. This book, or parts thereof, may not be reproduced in any form or by any means, electronic or mechanical, including photocopying, recording or any information storage and retrieval system now known or to be invented, without written permission from the Publisher.

ISBN 981-02-0498-1

Printed in Singapore by Utopia Press. 


\section{Preface}

In this book, we describe in some detail a rather unique algebraic approach to elementary particle physics which has been developed by the authors and their collaborators in the past two decades.

In elementary particle physics, there are a number of well recognized underlying symmetries which correctly predict the spectacular multiplet structures of observable particles. However, lack of a consistent method to deal with badly broken symmetries has considerably hindered us from investigating the dynamics of particle physics from symmetry point of view. Group theoretical method is usually based on the notion of exact symmetry.

With this book, however, we wish to arouse interest in this not well addressed important problem, by describing our point of view, method and its consequences in comparison with experiment.

We describe a formalism in which we deal directly only with physical particles but not virtual particles. We argue that the spectrum generating symmetries, though broken, still maintain their original linearities in a particular way, called asymptotic symmetry, which is, however, valid only for the physical particle multiplet with infinite momentum.

When combined with the presence of the equal-time commutation relations involving the generators of underlying symmetry group, which are still valid in broken symmetry, asymptotic symmetry then immediately produces constraints on physical particle spectrum, displaying a close interplay among the masses, mixing parameters and coupling constants. This is the salient feature of the approach.

In this book we pursue the scenario described above without using the Feynman diagrams which involve virtual particles. Therefore, the method is in principle non-perturbative and deals directly with observable particles. We nevertheless show that we can reproduce the successful result of naive quark model and improve the shortcoming of the non-relativistic aspects of the model. We can also trace the origin of the observed remarkable mass and mass-mixing-angle relations in QCD and electroweak theory. We also derive many of the successful results of old $S U(6)$ symmetry without assuming $S U(6)$ symmetry and simultaneously recognize the presence of the dynamical selection rules observed within the theoretical frame- 
work, without imposing them from outside. We can thus essentially count quarks and anti-quarks algebraically, without really seeing them.

We are beginning to notice that one can approach particle spectroscopy also from a point of view of asymptotic $S U(2)_{L} \times U(1)_{Y} \times S U(3)_{\text {family }}$ symmetry. The method may even give us a powerful tool for the study of new physics where fundamental Lagrangian is not yet known.

This book is thus written with a spirit that in the quest of unknown, one should not be confined too rigidly to traditional thinking or method and there might be a way to approach broken symmetry in a non-perturbative way. Otherwise, the beautiful group theory remains to be a mere mathematical device to classify multiplicity in the symmetry limit. As will often be mentioned in the book, many difficult problems remain, of course, unresolved or untouched and await for fresh ideas and innovations of the method.

The book was planned to be readable for the physicists who know the basics of the concepts of quantum field theory. In Chapter 2, we give for non-experts a quick but hopefully sufficient review of the present picture of elementary particle physics and lay out the problems which have given motivations for the approach pursued in the later chapters of this book.

The project which started near the end of the sixties has evolved, along with the amazing experimental and theoretical development of elementary particle physics, gradually into a more ambitious undertaking, thanks to the efforts of many active collaborators and supports.

One of the authors (Sadao Oneda) owes his first training and introduction to the exciting world of particle physics to his outstanding teacher and dedicated physicist, Professor Shoji Ozaki.

Enthusiastic collaboration and pioneering effort of Dr. Seisaku Matsuda in the early stage was indispensable and is greatly appreciated. Professor Hiroomi Umezawa has always been a mentor and a collaborator on the pursuit of the idea related to asymptotic symmetry.

Many former students of S. Oneda have completed their Ph.D. thesis at the University of Maryland and many visitors to the University have collaborated with $\mathrm{S}$. Oneda on various aspects and phases of the development of the present approach. Many of their contributions are included in the book. He also thanks his old and new colleagues at the University of Maryland for discussions, advice and, above 
all, keeping an ideal environment for creative research.

$\mathrm{S}$. Oneda acknowledges the support from various sources on this project in the past: the National Science Foundation, center for Theoretical Physics and General Research Board of the University of Maryland, Japanese Ministry of Education for supporting many visiting collaborators, Toray Science Foundation and Suga Gijutsushinko Foundation of Japan. He also acknowledges the support and hospitality of the Research Institute for Fundamental Physics (now Yukawa Institute for Theoretical Physics), Kyoto University, where many of the collaborations were started. He is especially indebted to Professor K. Nishijima and Professor Z. Maki.

Y. Koide acknowledges the generous support of Shizuoka Prefectural Government Grant and the hospitality of the Elementary Particle Physics Group at University of Maryland. He also thanks Dr. K. Terasaki and Dr. M. Yasuè for useful discussions on the related subjects.

Both of the authors thank Professor Milton D. Slaughter for going over the manuscript and providing valuable comments. They also thank Professor H. Fusaoka for his contribution to Chapter 5 .

The authors thank editors of World Scientific, Ms. Frances Pordes, Ms. Y. M. Chong and Ms. Juliana Teoh, for their timely invitation to write this book and their warm cooperation.

The manuscript of this book was provided by the computer typesetting system TEX. The authors thank E. Watanabe, C. Nakata, H. Nakayasu, K. Yokoyama and $\mathrm{K}$. Hongou for typesetting this manuscript onto the Latex file.

Finally, but not the least, both of the authors thank their families and friends for their cheerful support.

Sadao Oneda

Yoshio Koide

April, 1991 
This page is intentionally left blank 


\section{Contents}

Preface $\mathrm{v}$

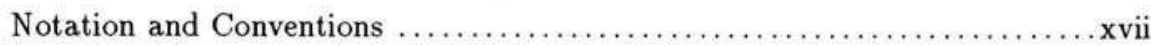

Chapter 1.

THE PURPOSE AND OUTLINE OF THIS BOOK $\ldots \ldots \ldots \ldots \ldots \ldots \ldots \ldots \ldots$

1.1 Why The Algebraic Approach? . ................................ 2

1.1.1 What is the algebraic approach? ............................

1.1.2 Historical development and new perspective $\ldots \ldots \ldots \ldots \ldots \ldots \ldots \ldots$

1.2 What is Asymptotic Symmetry?

1.2.1 Underlying broken symmetries in elementary particle physics $\ldots \ldots \ldots 6$

1.2.2 Constraining algebras related to underlying symmetry $\ldots \ldots \ldots \ldots \ldots 6$

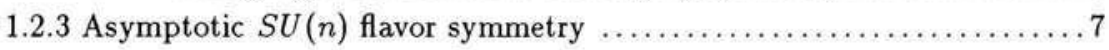

1.2.4 Hadron pattern recognition through asymptotic symmetry $\ldots \ldots \ldots \ldots .9$

1.2.5 Level realization of asymptotic flavor symmetry in chiral algebras . . . 10

1.3 Can the Algebraic Approach be Extended to the New Physics? ............11

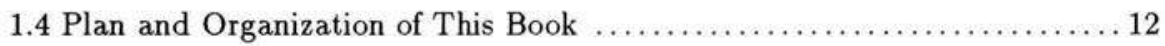

Chapter 2.

PARTICLES AND INTERACTIONS - A REVIEW .................. 16

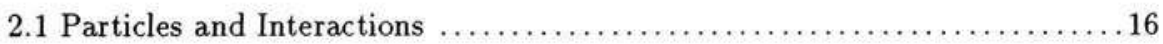

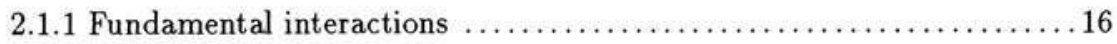

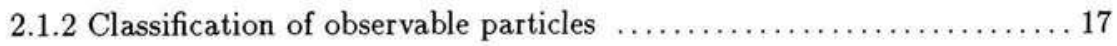

2.1.3 $S U(3)$ multiplets of the old hadrons $\ldots \ldots \ldots \ldots \ldots \ldots \ldots \ldots \ldots \ldots \ldots \ldots$

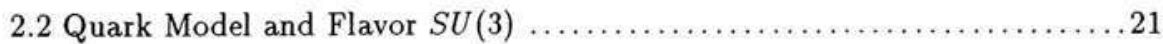

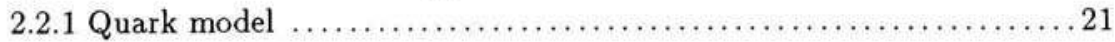

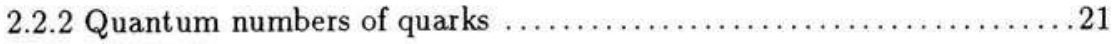

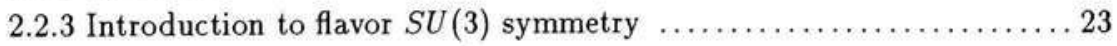

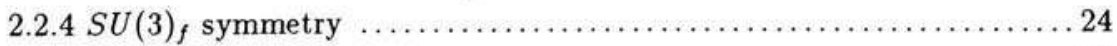

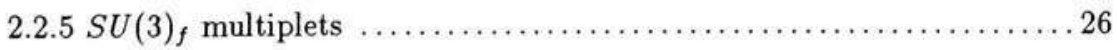

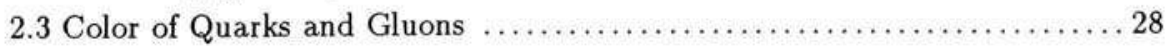

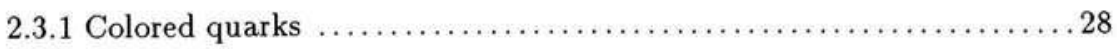

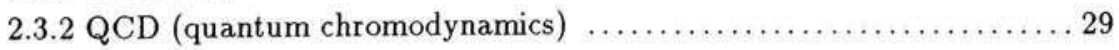


2.4 More Quarks and Classification of Hadrons

2.4.1 More quarks

2.4.2 Naming scheme for hadrons

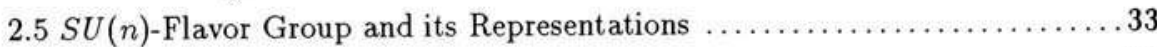

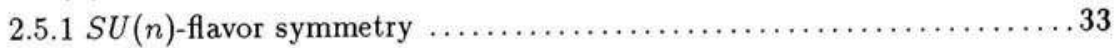

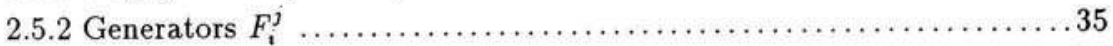

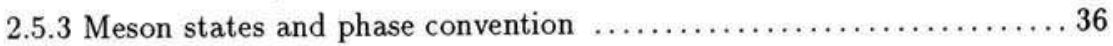

$2.6 S U(n)$ Representations and Young Tableaux $\ldots \ldots \ldots \ldots \ldots \ldots \ldots \ldots \ldots \ldots \ldots$

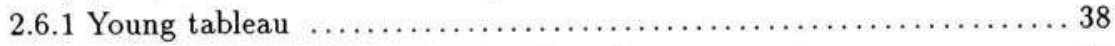

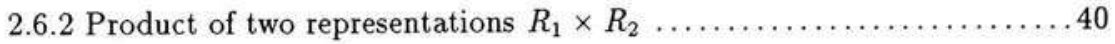

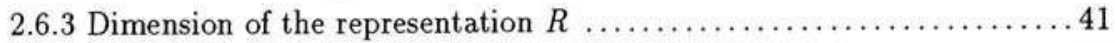

2.6.4 Decomposition of $S U(m+n)$ into $S U(m) \times S U(n) \ldots \ldots \ldots \ldots \ldots 42$

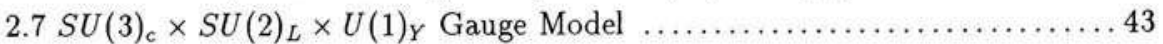

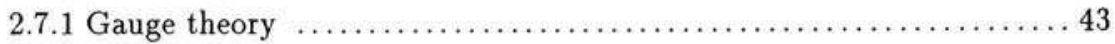

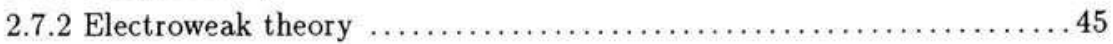

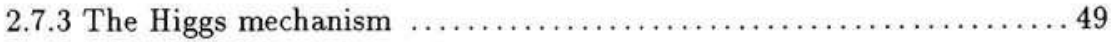

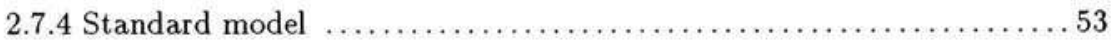

Chapter 3.

CURRENTS, CHARGES AND COMMUTATORS - A REVIEW .........56

3.1 Currents and Their Divergences in Broken Internal Symmetries ......... 56

3.1.1 Gell-Mann-Levy method in broken symmetry ................ 56

3.1.2 Example 1: $S U(3)_{f}$ invariant free quark Lagrangian $\ldots \ldots \ldots \ldots \ldots \ldots 57$

3.1.3 Example 2: Broken $S U(3)_{f}$ free quark Lagrangian .............. 58

3.1.4 Chiral transformation in broken $S U(3)_{f}$ free quark Lagrangian .....6 60

3.2 Underlying Chiral $S U(n)_{f L} \times S U(n)_{f R}$ Symmetry in QCD $\ldots \ldots \ldots \ldots \ldots 61$

3.3 Hadron Constraining $S U(n)_{f L} \times S U(n)_{f R}$ Algebras $\ldots \ldots \ldots \ldots \ldots \ldots 67$

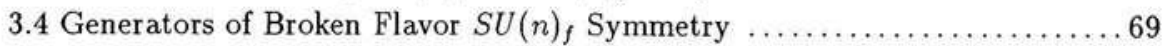

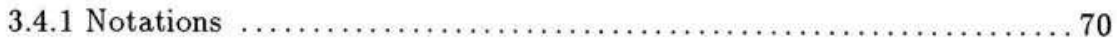

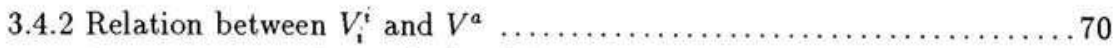

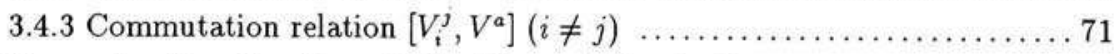

3.5 Symmetry Breaking Prescription Through Exotic Commutators ......... 72

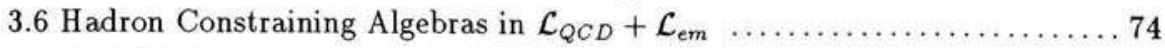

3.6.1 Electromagnetic currents ............................ 74 
Chapter 4.

ASYMPTOTIC BEHAVIOR OF BROKEN FLAVOR SYMMETRY $\ldots \ldots \ldots 78$

4.1 Pattern Recognition in Broken Symmetry ...................... 78

4.2 Simple Flavor Mass Splittings of Hadron Multiplets ................. 78

4.3 Another Effect of Symmetry Breaking - Particle Mixing .............. 80

4.4 Formulation of Asymptotic Flavor Symmetry .................... 84

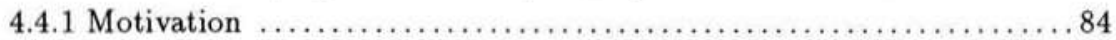

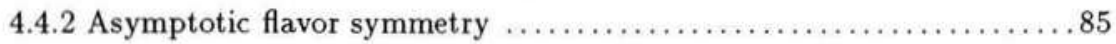

4.4.3 Vacuum annihilation in broken flavor symmetry $\ldots \ldots \ldots \ldots \ldots \ldots . \ldots 87$

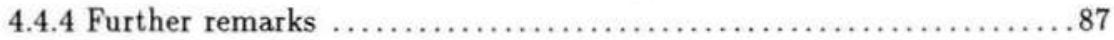

4.5 Implication for the Matrix Elements of $S U(n)_{f}$ Generators ............ 88

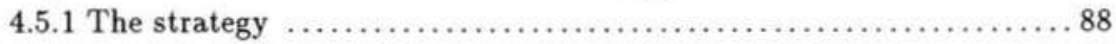

4.5.2 Explicit implication of asymptotic flavor symmetry ............. 89

4.5.3 Phenomenological implication of asymptotic symmetry ............ 90

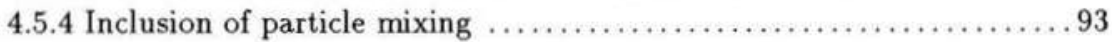

4.5.5 Ademollo and Gatto theorem ............................ 95

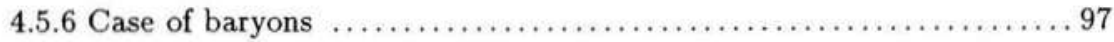

4.5.7 Summary: Asymptotic parametrization of the matrix elements of

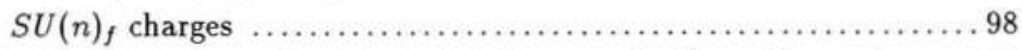

4.6 Mass Splitting Patterns Consistent with Asymptotic Flavor Symmetry ....99

4.6.1 Argument for asymptotic flavor symmetry ................... 99

4.6.2 Derivation of remarkable simple mass relations in $S U(n)_{f} \ldots \ldots \ldots .99$

4.6.3 Prediction on $1^{--} \rightarrow 0^{-+}$meson radiative transitions $\ldots \ldots \ldots \ldots \ldots 103$

4.6.4 Why can some of the results based on asymptotic $S U(4)_{f}, S U(5)_{f}$

be so good? ....................................... 104

4.6.5 Derivation of dynamical selection rule

- Presence of the quark-line rule in the formalism .............. 105

4.7 Asymptotic Behavior of the Matrix Elements of Axial-Vector Charge $A^{a} .107$

4.7.1 $S U(n)_{f}$ parametrizations of the asymptotic matrix elements

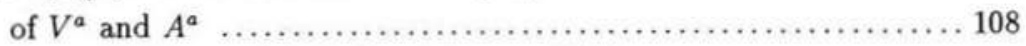

4.7.2 Important implications of asymptotic $S U(3)_{f}$ symmetry

- Cabibbo sum rules can be exact sum rules! 
4.8 PCAC and Hard-PS-Meson Extrapolation $\ldots \ldots \ldots \ldots \ldots \ldots \ldots \ldots \ldots \ldots \ldots \ldots \ldots \ldots$

4.8.2 PCAC and hard PS-meson extrapolation for the asymptotic

hadron matrix elements of axial-vector charges $\ldots \ldots \ldots \ldots \ldots \ldots 116$

4.8.3 Broken $S U(n)_{f}$ symmetry relations for the $B \rightarrow B^{\prime}+$ PS-meson

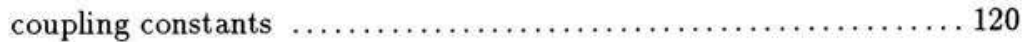

4.8.4 Broken $S U(3)_{f}$ in the scattering processes involving PS-mesons . ....129

4.8.5 Comment on hard $K$-meson, $D$-meson, $\cdots$ extrapolation $\ldots \ldots \ldots \ldots 130$

Chapter 5 .

ON THE FOUNDATION OF ASYMPTOTIC FLAVOR SYMMETRY $\ldots \ldots 131$

5.1 A Heuristic Model $-S U(2)_{I}$ Triplet and Singlet Spin 0 System ........ 131

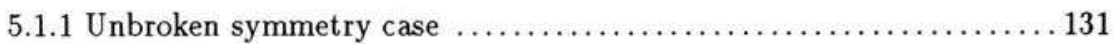

5.1.2 Broken symmetry and the Bogoliubov transformation ........... 134

5.1.3 Another method to derive the Bogoliubov transformation ........ 137

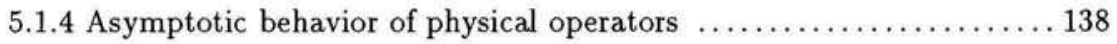

5.1.5 Derivation of mass-mixing angle constraint $\ldots \ldots \ldots \ldots \ldots \ldots \ldots \ldots$

5.1 .6 Additional remarks .................................... 143

5.2 Asymptotic Flavor Symmetry - The Boson Case ................. 144

5.2 .1 A derivation of asymptotic $S U(3)_{f}$ symmetry ................ 144

5.2.2 Derivation of the Gell-Mann-Okubo mass formula with mixing . . . 149

5.3 Asymptotic Flavor Symmetry - The Baryon Case ................ 150

5.3.1 Asymptotic relations for Dirac particles .................... 151

5.3.2 A simple example of $Z$-diagram effects .................... 155

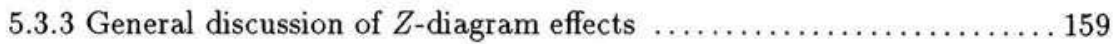

5.3.4 SU(3)-flavor mass formulas for baryons .................... 165

5.3.5 Further comments on ground-state hadrons in broken $S U(3)_{f}$

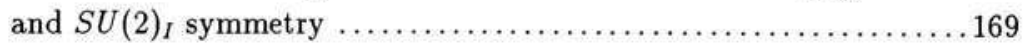

Chapter 6

HADRON SPECTROSCOPY THROUGH CONSTRAINING ALGEBRAS AND

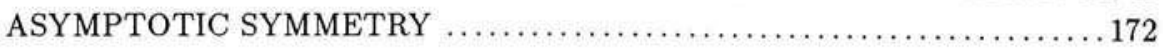

6.1 Exotic C.R.'s and Resulting Multiplet Mass Relations ............... 172

6.2 More Realistic Hadron Spectroscopy in QCD ........................ 175 


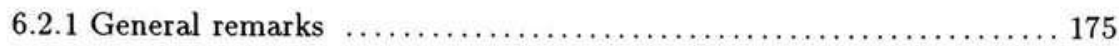

6.2.2 Extension to $S U(4)_{f}-$ Charmonium ....................... 176

6.3 Mass-Quark Structure-Coupling Constant Interplay $\ldots \ldots \ldots \ldots \ldots \ldots \ldots$

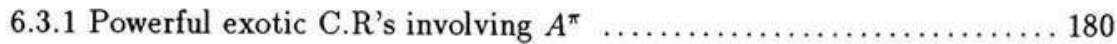

6.3.2 Simple inter-multiplet mass relations $\left(C_{t} C_{u}=-1\right.$ sum rules $) \ldots \ldots 180$

6.3.3 Selection rules for transitions between levels ................. 182

6.3.4 $S U(n)_{f}$ leakage in the same level excitation ................... 185

6.3.5 Mass-mixing angle-coupling constant interplay $\left(C_{t} C_{u}=1\right.$ sum rules) 185

$6.4 q \bar{q}$-Meson Mixing in the Strange, Charm, $\cdots$ Sector $\ldots \ldots \ldots \ldots \ldots \ldots . \ldots 196$

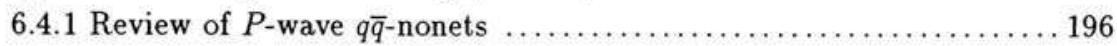

6.4.2 Correlation between $1^{++}$and $1^{+-}$mesons ..................... 198

$6.5 \Gamma\left(\tau \rightarrow K^{*} \nu_{\tau}\right) / \Gamma\left(\tau \rightarrow \rho \nu_{\tau}\right)$ and $V \rightarrow \bar{\ell} \bar{\ell}$ Decay Sum Rules $\ldots \ldots \ldots \ldots . .206$

6.5.1 Prediction on the $\tau \rightarrow \rho \nu_{\tau}$ and $K^{*} \nu_{\tau}$ branching ratio ..........206

6.5.2 Sum rules for vector mesons $\rightarrow \bar{\ell} \bar{\ell}$ decays $\ldots \ldots \ldots \ldots \ldots \ldots \ldots . \ldots 20 . \ldots \ldots$

6.6 PS-Meson Decay Constants and Inter-Multiplet Mixings ...............213

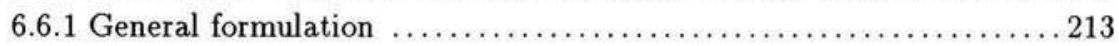

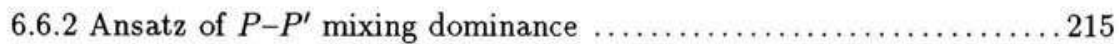

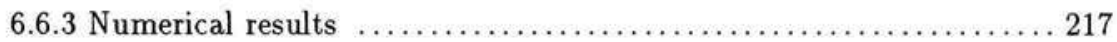

6.7 Glueballs and Their Effects on Meson Spectroscopy ................ 219

6.7.1 Glueballs as legitimate members of hadrons ................. 219

6.7.2 Glueballs and the violation of quark-line rule $\ldots \ldots \ldots \ldots \ldots \ldots \ldots 219$

6.7.3 Does $\eta^{\prime}(958)$ contain a sizable glue component? ................220

6.8 PCAC Sum Rules Involving Mesons and Vacuum .................. 229

Chapter 7.

LEVEL REALIZATION OF ASYMPTOTIC FLAVOR SYMMETRY IN CHIRAL ALGEBRAS (POSSIBLE ALTERNATIVE TO $\left.S U(6)_{W}\right) \ldots \ldots \ldots \ldots \ldots \ldots 235$

7.1 How to Deal with the Axial-Vector Charge? ................... 235

7.1.1 Historical development (Adler-Weisberger sum rule) $\ldots \ldots \ldots \ldots \ldots 235$

7.1.2 Asymptotic $S U(n)_{f}$ symmetry contents of the constraining

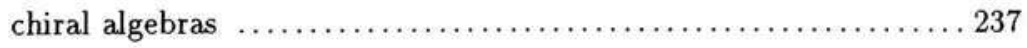

7.2 Level Scheme of Hadrons and its Possible Role in Realization ...........237

7.3 Formulation of Level Realization in the Infinite Momentum Frame ......2239

7.4 Derivation of the Good Results of $S U(6)_{W}$ and the Quark-Line Rule ....241 
7.4.1 Pattern recognition from charge algebras $\left[A^{\pi^{+}}, A^{\pi^{-}}\right]=2 V^{\pi^{0}}$

$$
\text { and }\left[\left[A^{\pi^{+}}, A^{\pi^{-}}\right], A^{\pi^{+}}\right]=2 A^{\pi^{+}} \text {, etc. }
$$

7.4.2 Boson pattern recognition from the algebra

$$
\left[\left[J_{\mu}^{e m}(x), A^{\pi^{+}}\right], A^{\pi^{-}}\right]=2 j_{\mu}^{3}(x)
$$

7.4.3 Further baryon pattern recognition from the algebra

$$
\left[\left[J_{\mu}^{e m}(x), A^{\pi^{+}}\right], A^{\pi^{-}}\right]=2 j_{\mu}^{3}(x) \ldots
$$

7.4.4 A theoretical motivation for the level realization ansatz

7.4.5 Comment on approximate $|\Delta I|=1 / 2$ rule 265

\section{Chapter 8}

ASYMPTOTIC SYMMETRY IN THE STANDARD ELECTROWEAK THEORY $\ldots 268$

8.1 A Global $S U(2)_{L}^{\prime}$ Symmetry in the Standard $S U(2)_{L} \times U(1)_{Y}$ Model $\ldots .268$

8.1.1 Spontaneously broken symmetry and asymptotic symmetry .......268

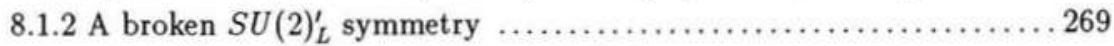

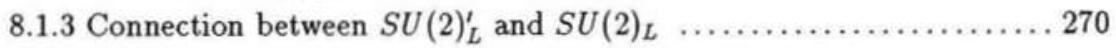

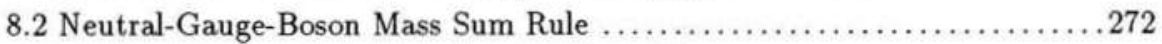

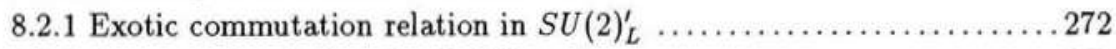

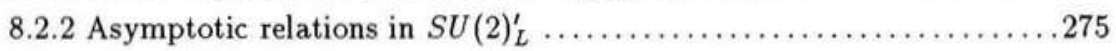

8.2.3 Mass-mixing angle sum rule from $\lambda= \pm 1$ components $\ldots \ldots \ldots \ldots 277$

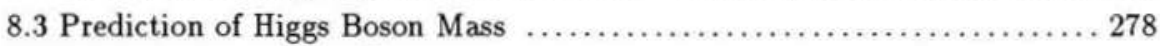

8.3.1 Asymptotic $W-\widehat{W}-\hat{\chi}$ relations ............................. 278

8.3.2 Elimination of unphysical operators $\hat{a}_{(0)}$ and $\hat{a}_{(3)} \ldots \ldots \ldots \ldots \ldots \ldots 280$

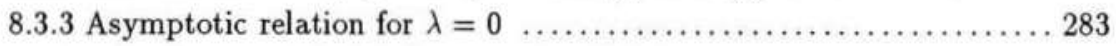

8.3.4 Remarks on the Higgs boson mass prediction $\ldots \ldots \ldots \ldots \ldots \ldots . \ldots 284$

Chapter 9

MESON MASSES AND QUARK-FAMILY MIXINGS IN BROKEN $S U(2)_{L} \times$

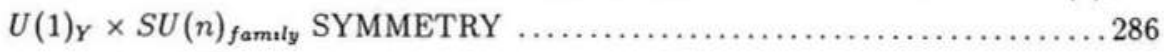

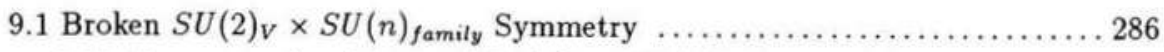

9.1.1 Symmetry breakdown of $S U(2)_{L} \times S U(2)_{R}$ into $S U(2)_{V} \ldots \ldots \ldots \ldots 286$

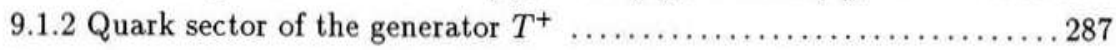

9.2 Derivation of Meson Mass-Quark Family Mixing Angle Sum Rule ...... 288

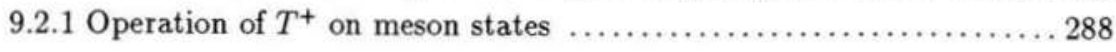




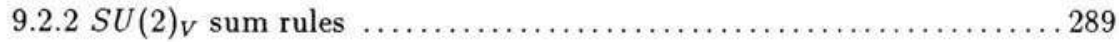

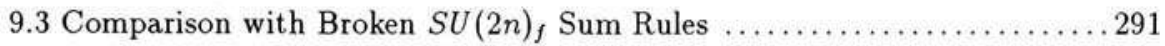

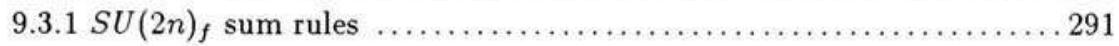

9.3.2 Number of the independent sum rules $\ldots \ldots \ldots \ldots \ldots \ldots \ldots \ldots \ldots 292$

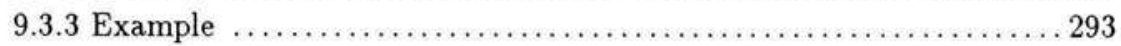

9.4 Sum Rules for the Cabibbo-Kobayashi-Maskawa Mixings

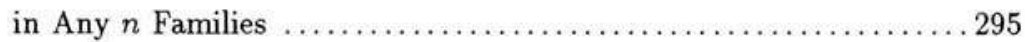

9.4.1 Sum rules for the CKM mixings ........................ 295

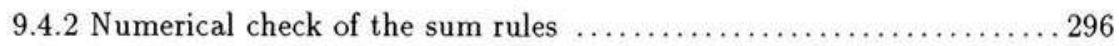

9.4.3 Possible extensions ....................................... 297

Chapter 10

FURTHER ATTEMPTS TOWARDS NEW PHYSICS ................. 301

10.1 Technicolored Bosons and Lepton Mass Relation in $S U(3)_{\text {family }} \ldots \ldots . . .301$

10.1.1 Technicolor scenario ................................... 301

10.1.2 Technicolored $S U(3)$-family $8+1$ boson model $\ldots \ldots \ldots \ldots \ldots \ldots 303$

10.1.3 A scenario of the $S U(3)$-family symmetry breaking ..............306

10.2 Gauge Boson Mass Sum Rules in Asymptotic SU(5) $)_{G U T}$ Symmetry ..... 308

10.2.1 Short review of the minimal $S U(5)_{G U T}$ model ................. 309

10.2.2 General formulation for $S U(N)$ gauge boson mass sum rules ......313

10.2.3 Higgs bosons and the surviving exotic C.R.'s ................... 316

10.2.4 $S U(5)_{\text {GUT }}$ gauge boson mass sum rules $\ldots \ldots \ldots \ldots \ldots \ldots \ldots \ldots \ldots \ldots \ldots \ldots \ldots$

Chapter 11

CONCLUDING REMARKS AND OUTLOOK $\ldots \ldots \ldots \ldots \ldots \ldots \ldots \ldots \ldots \ldots \ldots$

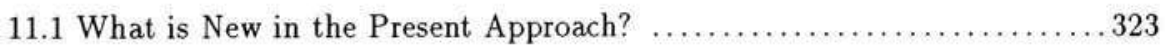

11.2 Future Tasks in Hadron and Quark-Lepton Physics

from Present Perspective ............................. 325

Appendix: Exotic Commutation Relations in Broken $S U(n)_{f}$ Symmetry .....331

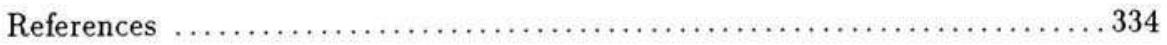

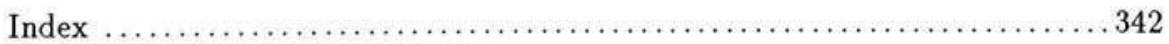


This page is intentionally left blank 


\section{Notations and Conventions}

\section{Metric and Related Definitions}

We denote the contravariant components of the four-dimensional coordinates $x^{\mu}$ as

$$
x^{\mu}=\left(x^{0}, x^{1}, x^{2}, x^{3}\right)=(t, x, y, z)=(t, \mathbf{r}),
$$

and the covariant components $x_{\mu}$ as

$$
x_{\mu}=g_{\mu \nu} x^{\nu}=(t,-\mathbf{r}) \text {, }
$$

where

$$
g_{00}=1, \quad g_{i j}=-\delta_{i j}, \quad g_{0 i}=0 \quad(i, j=1,2,3) .
$$

The gradient operators are expressed as

$$
\begin{aligned}
\partial^{\mu} & \equiv \frac{\partial}{\partial x_{\mu}}=\left(\frac{\partial}{\partial t},-\nabla\right), \\
\partial_{\mu} & \equiv \frac{\partial}{\partial x^{\mu}}=\left(\frac{\partial}{\partial t}, \nabla\right),
\end{aligned}
$$

where

$$
\nabla \equiv\left(\frac{\partial}{\partial x}, \frac{\partial}{\partial y}, \frac{\partial}{\partial z}\right)
$$

The scalar product of the two Lorentz four-vectors $A^{\mu}$ and $B^{\mu}$ is expressed as

$$
(A B)=A_{\mu} B^{\mu}=A_{0} B_{0}-\mathbf{A B} .
$$

The Levi-Civita symbol $\varepsilon^{\mu \nu \rho \sigma}$ is defined by

$$
\varepsilon^{0123}=-1 \text { i.e., } \varepsilon_{0123}=+1 .
$$

The $\gamma$ Matrices

The $\gamma$ matrices are defined as

$$
\begin{gathered}
\left\{\gamma^{\mu}, \gamma^{\nu}\right\} \equiv \gamma^{\mu} \gamma^{\nu}+\gamma^{\nu} \gamma^{\mu}=2 g^{\mu \nu}, \\
\gamma^{0 \dagger}=\gamma^{0}, \quad \gamma^{k \dagger}=-\gamma^{k} \quad(k=1,2,3),
\end{gathered}
$$


i.e.,

$$
\gamma^{\mu \dagger}=\gamma^{0} \gamma^{\mu} \gamma^{0}
$$

and

$$
\gamma_{\mu} \equiv\left(\gamma_{0}, \gamma_{k}\right)=\left(\gamma^{0},-\gamma^{k}\right) .
$$

The antisymmetric matrices $\sigma_{\mu \nu}$ are defined by

$$
\sigma_{\mu \nu}=\frac{i}{2}\left(\gamma_{\mu} \gamma_{\nu}-\gamma_{\nu} \gamma_{\mu}\right)
$$

which satisfy $\sigma_{\mu \nu}^{\dagger}=\sigma_{\mu \nu}$.

The $\gamma_{5}$ matrix is defined by

$$
\gamma_{5} \equiv \gamma^{5}=i \gamma^{0} \gamma^{1} \gamma^{2} \gamma^{3}
$$

so that it satisfies the relations

$$
\gamma_{5}^{\dagger}=\gamma_{5} \text { and } \gamma_{5}{ }^{2}=+1
$$

In our definition of $\gamma_{5}$, the helicity projection operators into the left-handed and right-handed states are given by $\frac{1}{2}\left(1-\gamma_{5}\right)$ and $\frac{1}{2}\left(1+\gamma_{5}\right)$, respectively:

$$
\psi_{L}=\frac{1}{2}\left(1-\gamma_{5}\right) \psi, \quad \psi_{R}=\frac{1}{2}\left(1+\gamma_{5}\right) \psi,
$$

where $\psi$ is four-component spinor. Therefore, the so-called " $V-A$ currents" are expressed by the form

$$
J_{\mu}^{V-A}(x)=\bar{\psi}(x) \gamma_{\mu}\left(1-\gamma_{5}\right) \psi(x)
$$

where $\bar{\psi}$ is defined by

$$
\bar{\psi}=\psi^{\dagger} \gamma_{0} .
$$

Sometimes, we use the following expression for a Lorentz scalar product with $\gamma_{\mu}$ :

$$
\begin{gathered}
\not A \equiv A_{\mu} \gamma^{\mu}=A_{0} \gamma_{0}-\mathbf{A} \boldsymbol{\gamma}, \\
\not \supset \equiv \gamma^{\mu} \partial_{\mu}=\gamma_{0} \partial_{0}+\boldsymbol{\gamma} \nabla .
\end{gathered}
$$




\section{$\underline{\text { Isospin Matrices }}$}

We will use $\tau^{a}$ matrices $(a=1,2,3)$ in the discussion of $S U(2)$ symmetry:

$$
\tau^{1}=\left(\begin{array}{ll}
0 & 1 \\
1 & 0
\end{array}\right), \quad \tau^{2}=\left(\begin{array}{cc}
0 & -i \\
i & 0
\end{array}\right), \quad \tau^{3}=\left(\begin{array}{cc}
1 & 0 \\
0 & -1
\end{array}\right),
$$

whose form is the same as the Pauli spin matrices $\sigma^{a}$

The following relations hold for the $\tau$ matrices:

$$
\begin{gathered}
\tau^{a} \tau^{b}-\tau^{b} \tau^{a}=2 i \epsilon_{a b c} \tau^{c}, \\
\tau^{a} \tau^{b}+\tau^{b} \tau^{a}=2 \delta_{a b}, \\
\operatorname{Tr}\left[\tau^{a} \tau^{b}\right]=2 \delta_{a b}, \\
\operatorname{Tr}\left[\tau^{a} \tau^{b} \tau^{c}\right]=2 i \epsilon_{a b c}, \\
\operatorname{Tr}\left[\tau^{a} \tau^{b} \tau^{c} \tau^{d}\right]=2\left(\delta_{a b} \delta_{c d}+\delta_{a d} \delta_{b c}-\delta_{a c} \delta_{b d}\right),
\end{gathered}
$$

where $\epsilon_{123}=+1$.

A scalar product $\boldsymbol{\tau} \pi$ with isospin triplet fields $\pi^{*}$ is expressed as

$$
\begin{gathered}
\boldsymbol{\tau} \boldsymbol{\pi} \equiv \sum_{a=1}^{3} \tau^{a} \pi^{a}=\sqrt{2}\left(\tau^{+} \pi^{+}+\tau^{-} \pi^{-}\right)+\tau^{3} \pi^{0} \\
=\left(\begin{array}{cc}
\pi^{0} & \sqrt{2} \pi^{+} \\
\sqrt{2} \pi^{-} & -\pi^{0}
\end{array}\right)
\end{gathered}
$$

where

$$
\begin{gathered}
\tau^{ \pm}=\frac{1}{2}\left(\tau^{1} \pm i \tau^{2}\right), \\
\pi^{ \pm}=\frac{1}{\sqrt{2}}\left(\pi^{1} \mp i \pi^{2}\right), \\
\pi^{0}=\pi^{3} .
\end{gathered}
$$

We will also denote $2 \times 2$ unit matrix as $\tau^{0}$;

$$
\tau^{0}=\left(\begin{array}{ll}
1 & 0 \\
0 & 1
\end{array}\right)
$$




\section{Normalization}

In the present book, the normalization of the state $|\mathbf{p}, \alpha\rangle$ is defined by

$$
\left\langle\mathbf{p}, \alpha \mid \mathbf{p}^{\prime}, \beta\right\rangle=\delta_{\alpha \beta} \delta\left(\mathbf{p}-\mathbf{p}^{\prime}\right)
$$

However, currently, various definitions of the normalization have been used. For convenience, we also list some examples of the general form when we define the normalization as

$$
\left\langle\mathbf{p}, \alpha \mid \mathbf{p}^{\prime}, \beta\right\rangle=\delta_{\alpha \beta} N_{\alpha} \delta\left(\mathbf{p}-\mathbf{p}^{\prime}\right) .
$$

The relation for creation and annihilation operators:

$$
\left[a_{\alpha}(\mathbf{p}), a_{\beta}^{\dagger}\left(\mathbf{p}^{\prime}\right)\right]=\delta_{\alpha \beta} \delta\left(\mathbf{p}-\mathbf{p}^{\prime}\right)
$$

or

$$
\left[a_{\alpha}(\mathbf{p}), a_{\beta}^{\dagger}\left(\mathbf{p}^{\prime}\right)\right]=\delta_{\alpha \beta} N_{\alpha} \delta\left(\mathbf{p}-\mathbf{p}^{\prime}\right) .
$$

The completeness integrals:

$$
1=|0\rangle\left\langle 0\left|+\sum_{\alpha} \int d^{3} p\right| \mathbf{p}, \alpha\right\rangle\langle\mathbf{p}, \alpha|,
$$

or

$$
1=|0\rangle\left\langle 0\left|+\sum_{\alpha} \int d^{3} p \frac{1}{N_{\alpha}}\right| \mathbf{p}, \alpha\right\rangle\langle\mathbf{p}, \alpha|
$$

Spinless field $\phi(x)$ :

$$
\phi(x)=\frac{1}{(2 \pi)^{3 / 2}} \int d^{3} p \frac{1}{\sqrt{2 E}}\left(e^{-i p x} a(\mathbf{p})+e^{i p x} a^{\dagger}(\mathbf{p})\right),
$$

or

$$
\phi(x)=\int d^{3} p \frac{1}{\sqrt{(2 \pi)^{3} 2 E N_{\phi}}}\left(e^{-i p x} a(\mathbf{p})+e^{i p x} a^{\dagger}(\mathbf{p})\right) .
$$

Matrix element of the bosonic vector currents:

$$
\left\langle\pi\left(\mathbf{p}^{\prime}\right)\left|V_{\mu}(0)\right| K(\mathbf{p})\right\rangle=\frac{1}{(2 \pi)^{3}} \frac{1}{\sqrt{4 E_{\pi} E_{K}}}\left[f_{+}\left(q^{2}\right)\left(p+p^{\prime}\right)_{\mu}+f_{-}\left(q^{2}\right)\left(p-p^{\prime}\right)_{\mu}\right],
$$

or

$$
\left\langle\pi\left(\mathbf{p}^{\prime}\right)\left|V_{\mu}(0)\right| K(\mathbf{p})\right\rangle=\frac{1}{(2 \pi)^{3}} \sqrt{\frac{N_{\pi}}{2 E_{\pi}}} \sqrt{\frac{N_{K}}{2 E_{K}}}\left[f_{+}\left(q^{2}\right)\left(p+p^{\prime}\right)_{\mu}+f_{-}\left(q^{2}\right)\left(p-p^{\prime}\right)_{\mu}\right],
$$


where $q=p^{\prime}-p$. Decay constant $f_{\pi}$ :

$$
\left\langle 0\left|A_{\mu}^{\pi^{+}}(0)\right| \pi^{+}(\mathbf{p})\right\rangle=\frac{1}{\sqrt{(2 \pi)^{3} 2 E}} i f_{\pi} p_{\mu},
$$

or

$$
\left\langle 0\left|A_{\mu}^{\pi^{+}}(0)\right| \pi^{+}(\mathbf{p})\right\rangle=\sqrt{\frac{N_{\pi}}{(2 \pi)^{3} 2 E}} i f_{\pi} p_{\mu} .
$$

For a Dirac field $\psi(x)$, we use the same normalization

$$
\left\{a^{(r)}(\mathbf{p}), a^{(s)}\left(\mathbf{p}^{\prime}\right)\right\}=\delta_{r s} \delta\left(\mathbf{p}-\mathbf{p}^{\prime}\right)
$$

as $(0.32)$, where

$$
\psi(x)=\frac{1}{(2 \pi)^{3 / 2}} \int d^{3} p \frac{1}{\sqrt{2 E}} \sum_{r=1,2}\left(a^{(r)}(\mathbf{p}) u^{(r)}(\mathbf{p}) e^{-i p x}+b^{(r) \dagger}(\mathbf{p}) v^{(r)}(\mathbf{p}) e^{i p x}\right),
$$

so that the normalizations for $u^{(r)}(\mathbf{p})$ and $v^{(r)}(\mathbf{p})$ must be

$$
u^{(r) \dagger}(\mathbf{p}) u^{(s)}(\mathbf{p})=v^{(r) \dagger}(\mathbf{p}) v^{(s)}(\mathbf{p})=2 E \delta_{r s},
$$

or

$$
\bar{u}^{(r)}(\mathbf{p}) u^{(s)}(\mathbf{p})=-\bar{v}^{(r)}(\mathbf{p}) v^{(s)}(\mathbf{p})=2 m \delta_{r}
$$

The projection operators for positive and negative energy states are given by

$$
\Lambda_{+}(\mathbf{p}) \equiv \sum_{r} u^{(r)}(\mathbf{p}) \bar{u}^{(r)}(\mathbf{p})=\not p+m,
$$

and

$$
\Lambda_{-}(\mathbf{p}) \equiv-\sum_{r} v^{(r)}(\mathbf{p}) \bar{v}^{(r)}(\mathbf{p})=-(\not p-m)
$$

respectively.

If we choose

$$
\left\{a^{(r)}(\mathbf{p}), a^{(s)}\left(\mathbf{p}^{\prime}\right)\right\}=N_{a} \delta_{r s} \delta\left(\mathbf{p}-\mathbf{p}^{\prime}\right)
$$

and

$$
\psi(x)=\frac{1}{(2 \pi)^{3 / 2}} \int d^{3} p \frac{1}{N_{\psi}} \sum_{r=1,2}\left(a^{(r)}(\mathbf{p}) u^{(r)}(\mathbf{p}) e^{-i p x}+b^{(r) \dagger}(\mathbf{p}) v^{(r)}(\mathbf{p}) e^{i p x}\right),
$$

then the normalizations for $u^{(r)}(\mathbf{p})$ and $v^{(r)}(\mathbf{p})$ must be

$$
u^{(r) \dagger}(\mathbf{p}) u^{(s)}(\mathbf{p})=v^{(r) \dagger}(\mathbf{p}) v^{(s)}(\mathbf{p})=\frac{\left|N_{\psi}\right|^{2}}{N_{a}} \delta_{r s},
$$


or

$$
\bar{u}^{(r)}(\mathbf{p}) u^{(s)}(\mathbf{p})=-\bar{v}^{(r)}(\mathbf{p}) v^{(s)}(\mathbf{p})=\frac{\left|N_{\psi}\right|^{2}}{N_{a}} \frac{m}{E} \delta_{r s} .
$$

The projection operators for positive and negative energy states are given by

$$
\Lambda_{+}(\mathbf{p}) \equiv \sum_{r} u^{(r)}(\mathbf{p}) \bar{u}^{(r)}(\mathbf{p})=\frac{\left|N_{\psi}\right|^{2}}{N_{a}} \frac{\not p+m}{2 E},
$$

and

$$
\Lambda_{-}(\mathbf{p}) \equiv-\sum_{r} v^{(r)}(\mathbf{p}) \bar{v}^{(r)}(\mathbf{p})=-\frac{\left|N_{\psi}\right|^{2}}{N_{a}} \frac{\not p-m}{2 E}
$$

respectively.

Although some of texts choose $N_{\psi}=\sqrt{E / m}$ or $E / m$, the choice is not useful for a treatment of massless Dirac particles. Therefore, in this book, we choose $N_{\psi}=\sqrt{2 E}$ and $N_{a}=1$ as well as those in boson fields. 


\section{Acronyms}

Certain acronyms used frequently are as follows:

C.G. coefficient Clebsch-Gordan coefficient

CKM mixing matrix Cabibbo-Kobayashi-Maskawa mixing matrix

C.R.

Commutation relation

CVC

Conserved vector current

ETC

Equal-time commutator

ETCR

Equal-time commutation relation

G-M-O mass formula

Gell-Mann-Okubo mass formula

GMOR

Gell-Mann-Oakes-Renner

GWS model

Glashow-Weinberg-Salam model

GUT

Grand unification theory

l.h.s.

left-hand side

L.S.Z. reduction

Lehmann-Symanzik-Zimmermann reduction

KSFR relation

Kawarabayashi-Suzuki-Fayyazuddin-Riazuddin relation

PCAC

PS-meson

Partially conserved axial-vector current

QCD

Pseudoscalar meson

QED

Quantum chromodynamics

r.h.s.

Quantum electrodynamics

VEV

right-hand side

Vacuum expectation value

\section{Experimental Data}

All experimental values referred in this book except for those with specific reference numbers are quoted from Particle Data Group, Review of Particle Properties, Phys. Lett. B239, 1 (1990), which is referred in this book as Ref. [0]. 